\title{
Oraler Eisen-Chelator kann die Ataxiesymptome nicht verbessern
}

Fragestellung: Kann Deferiprone, eine oral applizierte Substanz die Eisen bindet, die Symptome einer Friedreich'schen Ataxie positiv beeinflussen?

Hintergrund: Es liegen vielfältige Hinweise darauf vor, dass der Eisenstoffwechsel - insbesondere der Mitochondrien - bei Patienten mit Friedreich'scher Ataxie gestört ist. Auf molekularer Ebene kommt es zu einer Eisenakkumulation mit vielfältigen Auswirkungen auf den Energiestoffwechsel. Daher wurde vorgeschlagen, Substanzen die Eisen binden (Chelate), im Rahmen der Behandlung bei Patienten mit Friedreich'scher Ataxie einzusetzen.

Patienten und Methodik: Es handelt sich um eine multizentrische, in Europa und Kanada durchgeführte Studie, in der Patienten mit Friedreich'scher Ataxie untersucht wurden. Die Studie war randomisiert, doppelblind und placebokontrolliert. Insgesamt wurden 72 Patienten in die Studie eingeschlossen und in vier Therapiegruppen eingeteilt. Die Behandlung erfolgte mit $20 \mathrm{mg}, 40 \mathrm{mg}$ oder $60 \mathrm{mg} / \mathrm{kg}$ Körpergewicht Deferiprone pro Tag oder mit Placebo. Der primäre Endpunkt der Studie waren die Sicherheit und die Verträglichkeit. Sekundäre Endpunkte waren die neurologische Untersuchung mit etablierten

Pandolfo M, Arpa J, Delatycki $\mathrm{MB}$ et al. Deferiprone in Friedreich ataxia: a 6-month randomized controlled trial. Ann Neurol $2014 ; 76: 509-21$ Skalen wie die Friedreich Ataxia Rating Scale, die International Cooperative Ataxia Rating Scale (ICARS), der 9-Hole-Peg-Test, der Timed
25-Foot Walk und Low-Contrast Letter Acuity. Außerdem wurden die Alltgsaktivitäten (Activities of Daily Living, ADL) und die kardiale Funktion untersucht.

Ergebnisse: Das Kollektiv der 72 Patienten verteilte sich zu gleichen Anteilen auf erwachsene Patienten sowie Patienten unter 18 Jahren. Die Per-Protokoll-Population umfasste 45 Patienten. 18 dieser Patienten erhielten pro Tag $20 \mathrm{mg} / \mathrm{kg}$ Körpergewicht Deferiprone, 16 Patienten 40 mg/kg Körpergewicht Deferiprone und elf Patienten bekamen Placebo.

25 der 72 Patienten brachen die Studie vorzeitig ab, davon sechs von 17 Patienten unter Placebo und 19 von 55 Patienten unter Deferiprone. Die häufigsten Ursachen für Studienabbrüche waren Nebenwirkungen. Die 60-mg/kg-KörpergewichtDosis wurde nicht länger verwendet, nachdem es bei zwei Patienten zu einer Verschlechterung der Ataxie gekommen war. Bei einem Patienten kam es unter 20 mg/kg Körpergewicht pro Tag Deferiprone zu einer reversiblen Neutropenie. Bei den Patienten, die $20 \mathrm{mg} / \mathrm{kg}$ Körpergewicht Deferiprone pro Tag erhielten, kam es zu keinen Änderungen der klinischen Parameter und Skalen, bei den Patienten mit der höheren Dosis kam es zu einer Verschlechterung der Ataxie. Nur bei gering betroffenen $\mathrm{Pa}$ tienten ergab sich ein positiver Trend für die 20-mg/kg-Körpergewicht-pro-Tag-Dosis von Deferiprone.

Schlussfolgerungen: Deferiprone hat ein akzeptables Sicherheitsprofil bei der Behandlung von Patienten mit Friedreich'scher Ataxie, allerdings sind keine überzeugenden Therapieeffekte zu beobachten.

\section{- Kommentar von Hans-Christoph Diener, Essen}

\section{Dieser Ansatz kann wohl verlassen werden}

Der therapeutische Ansatz ist molekularbiologisch interessant und sinnvoll. Die vorliegende Studie ist eine der größten randomisierten prospektiven Studien zur Behandlung der Friedreich'schen Ataxie. Leider war Deferiprone nicht in der Lage, die Symptome der Friedreich'schen Ataxie positiv zu be-

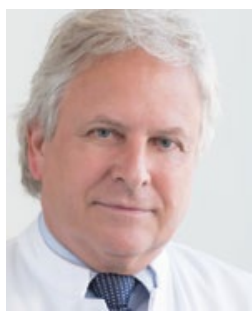

Prof. Dr. med. Hans-Christoph Diener, Essen

Direktor der Klinik für Neurologie,

Universitätsklinikum Essen

E-Mail:h.diener@uni-essen.de einflussen. Bei der höheren Dosis von Deferiprone ergab sich sogar ein Trend zugunsten einer Verschlechterung der Erkrankung. Ob die kleine Untergruppe von Patienten, die wenig betroffen war und möglicherweise ein Benefit hatte, eine größere erneute Studie wert ist, steht zu bezweifeln.

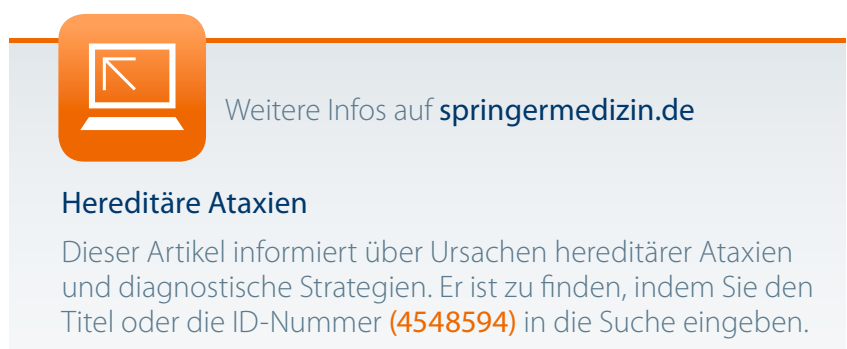

